\title{
Dispensación de fentanilo y tapentadol en farmacia comunitaria: adherencia farmacoterapéutica en dolor no oncológico
}

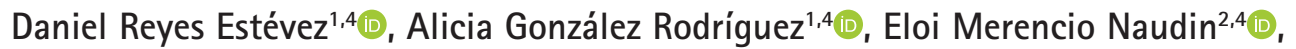 \\ $\mathrm{M}^{\mathrm{a}}$ Edelmira Córcoles Ferrándiz ${ }^{3,4}$ (1) \\ 1. Grupo de Dolor de SEFAC. 2. Coordinador del Grupo de Dolor de SEFAC en la fecha del estudio. 3. Coordinadora del Grupo de Dolor de SEFAC. \\ 4. Farmacéutico/a comunitario/a.
}

\section{PALABRAS CLAVE}

Analgésicos opioides, dolor crónico, cumplimiento y adherencia al tratamiento, farmacia comunitaria, farmacéutico comunitario, servicios profesionales farmacéuticos

\section{ABREVIATURAS}

AEMPS: agencia Española de Medicamentos y Productos Sanitarios

AF: atención farmacéutica

CRD: cuaderno de recogida de datos

DCNO: dolor crónico no oncológico

EPS: educación para la salud EVA: escala visual analógica FC: farmacéuticos comunitarios IPM: información personalizada del medicamento

OMS: Organización Mundial de la Salud

PNT: procedimiento normalizado de trabajo

PRM: problemas relacionados con el medicamento

RNM: resultados negativos de la medicación

SEFAC: Sociedad Española de Farmacia Clínica, Familiar y

Comunitaria

SEFV: Sistema Español de

Farmacovigilancia

SNC: sistema nervioso central

SPFA: servicio profesional

farmacéutico asistencial

\section{RESUMEN}

Introducción: durante los últimos años ha existido un aumento en la prescripción y dispensación de opioides y una elevada prevalencia de pacientes con dolor crónico no oncológico. Objetivo principal; evaluar adherencia farmacoterapéutica de pacientes con dolor no oncológico en tratamiento con fentanilo o tapentadol.

Material/Métodos: estudio observacional, descriptivo, transversal realizado por 139 farmacéuticos comunitarios. Población diana: mayores de 18 años que acuden a la farmacia comunitaria con prescripción de fentanilo o tapentadol. Las variables recogidas fueron edad, sexo, patología, carácter crónico/agudo, indicación, motivo del dolor, principios activos dispensados (conocimiento de posología, administración, posibles reacciones adversas), adherencia (Test de Haynes-Sackett), intensidad del dolor (Escala Visual Analógica), e intervención del farmacéutico.

Para medir la adherencia se utilizó el test de Haynes-Sackett, que permite detectar pacientes no adherentes, conocer las dificultades que tienen en el uso de fentanilo o tapentadol y ofrecer recomendaciones para mejorar el uso de estos tratamientos y minimizar los problemas de falta de adherencia.

La evaluación del dolor se realizó solo a los pacientes en tratamiento de continuación; determinando el valor medio mediante la medición a través de la EVA del dolor.

Resultados: el análisis de la adherencia con el test de Haynes-Sackett mostró que 358 pacientes $(87,1 \%)$ no tuvieron dificultad para tomarse/ponerse tratamiento, y 53 pacientes $(12,9 \%)$ sí presentaron dificultad, de los cuales, un $77 \%$ son mayores de 70 años y un $79 \%$ son mujeres.

Los principales motivos de la no adherencia en la población del estudio fueron: ser persona dependiente, tener dificultad en la colocación y tener problemas de adhesión del parche de fentanilo.

La evaluación media del dolor en los pacientes del estudio fue de 6,1 puntos en la EVA; un $44,5 \%$ de los pacientes presenta valores de EVA superior a 6 puntos, para los pacientes no adherentes el valor promedio de la EVA fue 6,6 mientras que para los pacientes adherentes fue 6 .

Para los pacientes con tratamiento crónico, refirieron un $41 \%$ tener el dolor controlado, $53 \%$ presentó mejora de su capacidad funcional y $57 \%$ mejora de su calidad de vida.

En un 37,9\% de los tratamientos de continuación se manifestaron reacciones adversas. La principal reacción adversa fue el estreñimiento presente en un 23,6\% de pacientes con tratamientos crónicos.

El 53,9\% de los pacientes respondió sí a querer un Servicio Profesional Farmacéutico Asistencial (SPFA) de abordaje del dolor crónico.

Conclusiones: la mayoría de pacientes son adherentes. El principal motivo de no adherencia fue ser una persona dependiente.

El valor promedio de EVA fue de 6 puntos. Los pacientes no alcanzan un control adecuado del dolor, aunque reconocen tener mejor capacidad funcional y calidad de vida.

En casi un 40\% de los tratamientos de continuación se manifestaron reacciones adversas.

La mitad de los pacientes demanda un servicio profesional farmacéutico asistencial en dolor.
Recibido: 20/2/2020

Aceptado: $11 / 6 / 2020$

Disponible online: 20/1/2021
Financiación: ninguna.

Conflicto de intereses: ninguno.

Cite este artículo como: Reyes D, González A, Merencio E, Córcoles ME. Dispensación de fentanilo y tapentadol en farmacia comunitaria: adherencia farmacoterapéutica en dolor no oncológico. Farmacéuticos Comunitarios. 2021 Jan 20; 13 (1): 32-59. doi:10.33620/FC.2173-9218.(2021/Nol13).001.06

Correspondencia: Daniel Reyes Estévez (leinad_77@hotmail.com).

ISSN 1885-8619 @SEFAC (Sociedad Española de Farmacia Clinica, Familiar y Comunitaria). Todos los derechos reservados. 


\section{KEYWORDS}

Analgesics opioid, chronic pain, treatment adherence and compliance, community pharmacy, community pharmacist, pharmaceutical services

\section{Dispensing of fentanyl and tapentadol in community pharmacy: Therapeutic adherence in non-cancer pain}

\section{ABSTRACT}

Introduction: In recent years there has been an increase on prescription and dispensation of opioids and high prevalence of patients with chronic non-oncological pain. Main objective; evaluate the pharmacotherapy adherence of patients with non-cancer pain treated with fentanyl or tapentadol.

Material/Methods: Observational, descriptive and transverse study performed by 139 community pharmacists. Target population: people over 18 years old that come to community pharmacy with a prescription of fentanyl or tapentadol. The collected variables were age, sex, pathology, chronic/acute character, indication, reason of pain, dispensed active principles (knowledge of posology, administration, possible adverse reactions), adherence (Haynes-Sackett Test), pain intensity (Visual Analog Scale), and the pharmacist's intervention.

Haynes-Sackett test was used to measure the adherence, which allows detecting non-adherent patients, knowing the difficulties related with the use fentanyl or tapentadol and offering recommendations to improve the use of these treatments and minimize the problems of lack of adherence.

The pain evaluation was carried out only in continuation care treatment; determining the average value using the VAS pain measuring system.

Results: The adherence analysis using Haynes-Sackett showed 358 patients (87.1\%) without difficulties to take/put on treatment while 53 patients $(12.9 \%)$ did present difficulties, of which 77\% were older than 70 years old and 79\% were women.

The main reasons for the non-adherence in the studied population were: being dependent people, having difficulties in the placement and having adhesion problems with fentanyl patches.

The average pain measure evaluation in the studied patients was 6.1 points in VAS; $44.5 \%$ of patients presented VAS values above 6 points, for the non-adherent patients the average VAS value was 6.6 while for the adherent patients was 6 .

In patients with chronic treatment, the 41\% had controlled pain, 53\% presented an improvement in their functional capacity and the 57\% had improved their quality life.

Adverse reactions were manifested in the $37.9 \%$ of the continuation care treatments. The main adverse reaction was the constipation, present in the $23.6 \%$ of the patients with chronic treatments.

53.9\% of the patients answered affirmative to be under Assistance Pharmaceutical Professional Service of boarding chronic pain.

Conclusions: Most of patients were adherent. The main reason for non-adherence was to be a dependent person.

VAS average value was 6 points and patients did not reach to an adequate pain control, although they recognize a better functional capacity and quality of life. Almost 40\% of the continuation care treatments manifested adverse reactions.

Half of the patients demanded Assistance Pharmaceutical Professional Service in pain.

\section{Introducción}

El dolor constituye una patología de importancia creciente a nivel mundial (1). Su prevalencia aumenta de forma paralela al envejecimiento de la población y cronificación de las enfermedades. Afecta a la salud física y psicológica y puede dar lugar a limitaciones funcionales en las personas que lo padecen, junto a un sufrimiento físico y emocional. Por ello, es tan importante conocerlo y poder medirlo de forma objetiva.

La medición objetiva es un proceso complejo, resulta difícil estimar, medir o valorar el dolor que siente una persona. Sin embargo, su cuantificación es muy importante y su evalua- ción es una actividad clave derivada de una intervención informativa y educativa para ayudar a mejorar la percepción que el paciente tiene sobre su dolor (2). Esto puede ayudar a que un dolor autolimitado y protector como es el dolor agudo, no pierda su esencia y no se mantenga en el tiempo convirtiéndose en un inútil y destructivo dolor crónico.

Las cifras de prevalencia de dolor crónico en la población general se encuentran entre el 15\% y el 25\% según datos basados en la población de Estados Unidos (3), cifra que aumenta al 50\% en los mayores de 65 años y en cualquier grupo afecta más a las mujeres que a los hombres.
En 2011, el Ministerio de Sanidad editó una publicación denominada "Unidad de tratamiento del dolor. Estándares y recomendaciones de calidad y seguridad". En ella, se recopilaron todos los estudios de prevalencia del dolor crónico desde el año 2001 (4), reflejando una prevalencia para el dolor crónico del 23,8\%, además de una relación mujer/hombre de $37 / 20$.

Según el estudio "Pain Proposal" en España, se estima que la prevalencia del dolor crónico se sitúa en torno al 17\% (5). Según el estudio de M Dueñas, la prevalencia del dolor crónico aumenta con la edad y es mayor en mujeres (6). 
Además, según dicho estudio en las mujeres es más frecuente el dolor en extremidades y/o articulaciones, mientras que en hombres es más frecuente el dolor de espalda (6).

En un $45,4 \%$ de la población con dolor crónico éste está presente en más de una localización, siendo más frecuente esta condición en la población de mayor edad y en las mujeres (6).

Por otro lado, la última Encuesta Nacional de Salud de España (2017) nos muestra la alta prevalencia de enfermedades que causan dolor crónico, entre las que destacan la lumbalgia, la artrosis y la cervicalgia (7).

Además, según los datos de la Encuesta Nacional de Salud de 2017 un 11,6\% de las personas que padecen dolor manifestaron que padecen dolor severo, un 64,1\% lo presentan con carácter moderado y un $24,1 \%$ indicaron que el dolor que presentan es leve (7).

Durante los últimos años, ha existido un aumento en la prescripción y dispensación de opioides, destacando fentanilo y tapentadol. El elevado número de pacientes con dolor crónico no oncológico, que acude a las farmacias comunitarias solicitando su dispensación, justifica el interés profesional.

Dentro del dolor crónico, el dolor crónico no oncológico (DCNO) supone un reto debido a su complejidad e imprevisibilidad de respuesta al tratamiento. Actualmente, aunque existe amplia bibliografía que recomienda el uso de opioides, tanto en dolores nociceptivos (8) como neuropáticos, solo existe amplia experiencia clínica en dolor nociceptivo, ya que en dolor neuropático existe una gran variabilidad de tipos de dolor y poca evidencia.

La efectividad a largo plazo de los opioides utilizados en el DCNO sigue siendo controvertida, ya que la mayoría de los estudios que hablan sobre su efectividad, no son superiores a 12 semanas de duración y los estudios sobre su seguridad no van más allá de 1 año (9), por lo que tienen que ser indicados y prescritos con precaución y siempre valorando a cada paciente de forma individual (10).
La efectividad inicial puede desaparecer aun siendo los pacientes adherentes al tratamiento debido a procesos farmacocinéticos, genéticos, etc. Al no tener techo de dosis, altas dosificaciones pueden originar efectos adversos propios de su uso y posología. Afectan al sistema nervioso central (somnolencia, mareos y sedación) y el aparato gastrointestinal (náuseas, vómitos $\mathrm{y}$ estreñimiento).

En los últimos años, ha habido un aumento en la prescripción y, como consecuencia, un crecimiento en la dispensación de fármacos opioides. Fentanilo y tapentadol son un ejemplo de ese aumento (11). Tanto es así que desde la Administración se están realizando programas de inspección para valorar el aumento de consumo de fentanilo.

En 2019 la Agencia Española de Medicamentos y Productos Sanitarios (AEMPS) emitió el informe "Utilización de medicamentos opioides en España durante el periodo 20102018" (12), donde se puede ver el aumento de consumo de todos los grupos de opioides, y tanto fentanilo como tapentadol tienen un aumento exponencial: 1,1 y 0,9 Dosis Diarias Definidas por 1.000 habitantes y día (DHD), respectivamente.

Cada vez se utiliza más este último escalón de la escalera analgésica clásica de la organización Mundial de la Salud (OMS) para tratar dolor crónico ya sea de origen oncológico o no oncológico (12).

En este entorno de práctica de la atención farmacéutica, el farmacéutico comunitario puede implicarse aún más evaluando la efectividad de los tratamientos y solucionando los problemas relacionados con el medicamento (PRM) y resultados negativos de la medicación (RNM), que identifique en su práctica profesional. En patologías crónicas que cursan con dolor crónico resulta conveniente que el farmacéutico comunitario pueda evaluar la respuesta terapéutica a los tratamientos en estos pacientes, y para esta valoración se debe tener en cuenta el grado de dolor.

La alta prevalencia de los pacientes aquejados de DCNO que acuden a las farmacias comunitarias justifica el interés profesional por ampliar conocimientos sobre el dolor crónico y sus tratamientos efectivos con el objetivo de poder abordarlos adecuadamente. Por ello, el farmacéutico comunitario tiene un papel muy importante en la dispensación de estos medicamentos, garantizando un uso responsable de los mismos.

Por último, y no menos importante, la actuación del farmacéutico en este ámbito no puede ser garantía de éxito si no abordamos el mayor problema que tienen nuestros pacientes, en su mayoría polimedicados: la adherencia farmacoterapéutica. Un problema, que si no es abordado y corregido puede ser motivo suficiente para el fracaso terapéutico.

¿Conocemos cuántos pacientes no usan los medicamentos como les fueron prescritos? ¿Qué dificultades tienen? ¿Cuál es la magnitud de la falta de adherencia farmacoterapéutica? Es conocido por algunos estudios realizados en pacientes crónicos que la falta de adherencia llega al 50\% (13), y se han llevado a cabo estudios de adherencia en pacientes asmáticos, psiquiátricos, hipertensos, diabéticos y en pacientes con dislipemias. Sin embargo, en pacientes con dolor crónico se ha investigado poco para conocer la tasa de falta de adherencia.

En paciente con DCNO en tratamiento con fentanilo o tapentadol es necesario poder detectar a los pacientes con falta de adherencia, e identificar las barreras que dificultan la adherencia y así seleccionar las estrategias adecuadas para mejorarla.

Se sabe que entre los principales factores para la no adherencia se encuentran: la complejidad de la terapia (múltiples dosis y fármacos), la presencia de efectos adversos, el riesgo percibido de la medicación y las creencias del paciente sobre su enfermedad, todos estos factores pueden tener una gran relevancia en los pacientes con dolor crónico no oncológico en tratamiento con fentanilo o tapentadol.

Los farmacéuticos comunitarios durante la dispensación facilitan información del proceso de uso de estos medicamentos, resolviendo posibles dudas respecto a la forma de administración, posología, duración del tratamiento, presencia de efectos 
adversos, al tiempo que detecta problemas de no adherencia (dificultades en la toma, desconocimiento del tratamiento, percepciones o creencias sobre este tipo de medicamentos...) y ofrecen las recomendaciones de mejora, así como la importancia de la adherencia farmacoterapéutica (14).

El trabajo que aquí se presenta analiza la adherencia farmacoterapéutica del paciente que utiliza estos analgésicos opioides para DCNO, así como la intensidad del dolor, las incidencias detectadas y tiene como objetivos:

\section{Objetivo principal}

- Evaluar la adherencia farmacoterapéutica de los pacientes con dolor no oncológico en tratamiento con fentanilo o tapentadol.

\section{Objetivos secundarios}

- Conocer las causas de falta de adherencia y las dificultades en el uso de estos tratamientos.

- Evaluar la intensidad del dolor con la EVA en los pacientes adherentes $\mathrm{y}$ no adherentes con dolor no oncológico.

- Conocer la percepción que tienen estos pacientes sobre la efectividad de su tratamiento con fentanilo o tapentadol.

- Describir los problemas relacionados con la medicación y las intervenciones llevadas a cabo durante la dispensación de estos tratamientos.

\section{Material y método}

La muestra utilizada para la realización de este trabajo (pacientes con dolor no oncológico) es una submuestra de pacientes que forma parte del estudio "Dispensación de fentanilo y tapentadol en farmacia comunitaria: perfil del paciente e indicación terapéutica en dolor crónico no oncológico" (15); estudio de corte descriptivo, observacional, transversal, multicéntrico y de ámbito nacional, realizado entre enero y marzo de 2018 por 139 farmacéuticos comunitarios españoles, e impulsado por el grupo de Dolor de la Sociedad Española de Farmacia Clínica, Familiar y Comunitaria (SEFAC), en el que se registraron 566 pacientes, de los que 559 (98,8\%) aceptaron participar en el estudio.

\section{Sujetos}

Población diana: mayores de 18 años que llegan a la farmacia comunitaria con prescripción de fentanilo o tapentadol.

\section{Criterios de inclusión}

Mayores de 18 años que aceptan participar y dan su consentimiento informado escrito.

\section{Criterios de exclusión}

Se excluyeron del estudio a menores de 18 años, personas con dificultad de comunicación y aquellos que no aceptaron participar.

\section{Desarrollo del estudio \\ Procedimiento}

Con el objetivo de servir como guía a todos los farmacéuticos participantes, previamente se les facilitó, a través de la plataforma online www. investigacionsefac.org, un procedimiento normalizado de trabajo (PNT): PNT Estudio 'Análisis del uso de Fentanilo y Tapentadol en farmacia comunitaria' (15), anexo 1.

Para facilitar su desarrollo, así como una normalización entre todos los investigadores a la hora de la recogida de datos, se proporcionó también a los farmacéuticos participantes, a través de la plataforma online www.investigacionsefac.org, el cuaderno de recogida de datos (eCRD): Registro de dispensación de opioides fuertes para dolor no oncológico (figura 1), específicamente elaborado para el estudio.

\section{Fase experimental}

Durante el periodo del estudio, en el momento de la dispensación, los farmacéuticos comunitarios cumplimentaron un e-CRD para cada paciente que permitió recoger información sobre el paciente, edad, sexo, patología, tiempo de evolución, indicación, motivo del dolor y principios activos dispensados. Además, se registraron otras variables para el estudio completo: conocimiento de posología, administración y posibles reacciones adversas, evaluación de adherencia (Test de Haynes-Sackett), dolor (Escala Visual Analógica -EVA), expectativas relacionadas con el tratamiento y la intervención del farmacéutico.
Para medir la adherencia se utilizó el test de Haynes-Sackett (16), que permite detectar pacientes no adherentes, conocer las dificultades que tienen en el uso de fentanilo o tapentadol y ofrecer recomendaciones para mejorar el uso de estos tratamientos y minimizar los problemas de falta de adherencia.

La evaluación del dolor se realizó utilizando la EVA (17-20). La valoración del grado de dolor permite evaluar la efectividad del tratamiento para el DCNO con dichos medicamentos y se realizó solo a los pacientes en tratamiento de continuación, determinando el valor medio mediante la medición a través de la EVA del dolor.

También se analizó la opinión personal del paciente con respecto a la eficacia de su tratamiento. Solamente a los pacientes con tratamientos de continuación se les preguntaba si pensaban que su dolor crónico estaba controlado, si había mejorado su capacidad funcional y si pensaban que había mejorado su calidad de vida.

Además, si el farmacéutico detectaba alguna incidencia, la estudiaba y evaluaba, identificando problemas relacionados con el medicamento (PRM) y resultados negativos de la medicación (RNM), clasificándolos según el listado propuesto por Foro de Atención Farmacéutica en su Documento de Consenso (21), procediendo a su registro, así como las intervenciones realizadas y el resultado en salud obtenido.

\section{Análisis de datos}

Una vez finalizado el período del estudio, se llevó a cabo el registro informático de los datos recogidos de todos los pacientes, y se procedió al análisis de los mismos. El tratamiento estadístico de los datos se realizó con una hoja de aplicación informática MSExcel ${ }^{\circledR}$.

Las variables cuantitativas se expresaron mediante las medidas de tendencia central y de dispersión (media y desviación estándar) y las variables cualitativas como frecuencias absolutas y relativas, incluyendo el intervalo de confianza al 95\%. Se utilizaron los test de la Chi cuadrado para las comparaciones de variables cualitativas y t de Student en las cuantitativas. Se consideró significativa una $\mathrm{p}<0,05$. 


\section{Resultados}

Se incluyeron 566 pacientes de los cuales 559 (98,8\%) aceptaron participar. De ellos $173(30,9 \%)$ fueron hombres y $386(69,1 \%)$ mujeres.

Se observó que fentanilo fue dispensado en $318(56,9 \%)$ casos y tapentadol en 241 (43,1\%). La dispensación de estos medicamentos fue diferente en función del sexo y edad del paciente, siendo significativo en el caso de la edad (tabla 1) (15).

La prescripción de estos opioides fue mayoritariamente para dolor no oncológico, fueron 478 pacientes (85,5\% IC95\% 82,6\% - 88,4\%), y dolor oncológico fueron 81 pacientes (14,5\% IC95\% 11,6\% - 17,4\%) (15).

En adelante el presente estudio se centrará en la submuestra de pacientes con dolor no oncológico constituida por 478 pacientes. Dentro de las prescripciones de dolor no oncológico, 459 (96,0\% IC95\% 94,3\% $97,8 \%$ ) fueron para DCNO (15).

Se evaluó la adherencia en los pacientes con dolor no oncológico en tratamiento de continuación con el test validado de Haynes-Sackett, 358 pacientes $(87,1 \%)$ no refirieron dificultad para tomarse/ponerse su tratamiento; al contrario, 53 pacientes $(12,9 \%)$ de los pacientes con tratamiento de continuación sí refirieron tener dificultad para tomarse/ponerse su tratamiento (no adherencia). Entre los pacientes con problemas de adherencia, según su edad, un $77 \%$ eran mayores de 70 años y, según su sexo, 79\% eran mujeres.

Observando la adherencia según el tipo de opioide empleado, resultó una tasa de no adherencia del 16\% para los pacientes en tratamiento con fentanilo para dolor crónico no oncológico, y solo un $6 \%$ de no adherencia en los pacientes tratados con tapentadol para dolor no oncológico, considerando no adherentes los que a la pregunta del test de Haynes-Sackett respondieron que sí (tabla 2).

Con respecto a los pacientes tratados con fentanilo que refirieron sí tener dificultad para tomarse/ponerse su tratamiento, hay que especificar que solamente presentaron dificultad en la vía transdérmica (tabla 3).

Los principales motivos de la no adherencia en la población del estudio fueron: ser persona dependiente, tener dificultad en la colocación y tener problemas de adhesión del parche de fentanilo (tabla 4).

La evaluación media del dolor en los pacientes del estudio fue de 6,1 puntos en la EVA; un 44,5\% de los pacientes presenta valores de EVA superior a 6 puntos. Mientras que si analizamos la intensidad del dolor teniendo en cuenta la adherencia, el valor promedio de la EVA en los pacientes no adherentes fue 6,6 puntos. Los pacientes adherentes obtuvieron un valor de EVA de 6 (tabla 5).
En cuanto a la percepción de la eficacia del tratamiento, los pacientes con dolor no oncológico consideran sí tener el dolor controlado en un $41 \%$, para un 53\% sí ha mejorado su capacidad funcional y para un $57 \%$ sí se ha mejorado su calidad de vida (tabla 6).

Respecto a las incidencias detectadas por los farmacéuticos comunitarios, además de los problemas de adherencia descritos anteriormente, 157 pacientes refirieron presentar reacciones adversas a los medicamentos (RAM) de los 414 pacientes con tratamientos de continuación para dolor no oncológico. Las principales reacciones adversas fueron: el estreñimiento presente en 98 pacientes (un 23,6\% de los tratamientos crónicos), sedación manifestada por 38 pacientes (un 9,1\% de los tratamientos crónicos), náuseas y/o vómito referida por 29 pacientes (un 7\% de los tratamientos crónicos) y prurito presente en 17 pacientes (un 4,1\% de los tratamientos de continuación).

En cuanto a la actuación del farmacéutico, los farmacéuticos participantes podían realizar más de una intervención si se valoraba necesario, resultando que la actuación mayoritaria fue la de dispensar con información sobre el medicamento en el $66,7 \%$ y en segundo lugar dispensar con educación para la salud 52,7\%. El resto de actuaciones están recogidas en la (tabla 7).

Tabla 1 Medicamento dispensado según el sexo y la edad del paciente (15)

\begin{tabular}{|l|c|c|c|c|c|c|}
\hline & Fentanilo & Tapentadol & Total & Fentanilo & Tapentadol & Ambos \\
\hline Hombre & 109 & 64 & 173 & $63 \%$ & $37 \%$ & $100 \%$ \\
\hline Mujer & 209 & 177 & 386 & $54 \%$ & $46 \%$ & $100 \%$ \\
\hline Total & 318 & 241 & 559 & & \multicolumn{2}{|c|}{$p=0,0505$} \\
\hline (18 a 39 años) & 9 & 9 & 18 & $50 \%$ & $50 \%$ & $100 \%$ \\
\hline (40 a 69 años) & 115 & 119 & 234 & $49 \%$ & $51 \%$ & $100 \%$ \\
\hline (>70 años) & 194 & 113 & 307 & $63 \%$ & $37 \%$ & $100 \%$ \\
\hline Total & 318 & 241 & 559 & & $p=0,0040$ & \\
\hline
\end{tabular}

Tabla 2 Adherencia farmacoterapéutica (Test de Haynes-Sackett) en pacientes con dolor no oncológico según el tipo de tratamiento

\begin{tabular}{|l|c|c|c|}
\hline Haynes-Sackett & Fentanilo & Tapentadol & Fentanilo \\
\hline Tratamiento de inicio. No se les pregunta & 23 & 44 & $9 \%$ \\
\hline Tratamiento continuación. Si se les pregunta & 235 & 176 & $91 \%$ \\
\hline Responden No & 195 & 163 & $83 \%$ \\
\hline Responden Si & 40 & 13 & $17 \%$ \\
\hline
\end{tabular}


Tabla 3 Adherencia farmacoterapéutica (Test Haynes-Sackett) en pacientes con dolor no oncológico en tratamiento con fentanilo según la vía de administración

\begin{tabular}{|l|c|c|c|}
\hline Via de administración del fentanilo & No & Si & $\begin{array}{c}\text { Tratamiento de inicio. } \\
\text { No se les pregunta }\end{array}$ \\
\hline Bucal & 13 & & 1 \\
\hline Intranasal & 1 & & \\
\hline Transdérmica & 170 & 40 & \\
\hline Transdérmica y bucal & 3 & & \\
\hline Transdérmica y transmucosa & 2 & & \\
\hline Transmucosa & 4 & & \\
\hline
\end{tabular}

Solo se incumple con la vía transdérmica.

Tabla 5 Promedio de la EVA según la adherencia farmacoterapéutica en pacientes con dolor no oncológico

\begin{tabular}{|l|c|c|c|}
\hline Haynes-Sackett & $\begin{array}{c}\text { Promedio de } \\
\text { la EVA }\end{array}$ & $\begin{array}{c}\text { Desviación } \\
\text { estándar }\end{array}$ & \\
\hline $\begin{array}{l}\text { Tratamiento de inicio. No se les } \\
\text { pregunta }\end{array}$ & 6,77 & 1,76 & \\
\hline $\begin{array}{l}\text { Tratamiento de continuación } \\
\text { respuesta No }\end{array}$ & 6,01 & 2,40 & \\
\hline $\begin{array}{l}\text { Tratamiento de continuación } \\
\text { respuesta Si }\end{array}$ & 6,60 & 2,34 & $p=0,097$ \\
\hline
\end{tabular}

Tabla 4 Causas de falta de adherencia farmacoterapéutica en pacientes con dolor no oncológico (Haynes-Sackett)

\begin{tabular}{|l|c|}
\hline Qué dificultad tiene & $\begin{array}{c}\text { No de } \\
\text { pacientes }\end{array}$ \\
\hline Fentanilo & 9 \\
\hline Al colocarlo & 2 \\
\hline Dudas de manejo & 1 \\
\hline Incumple voluntariamente & 7 \\
\hline Mala adhesión del parche & 17 \\
\hline Necesita ayuda & 2 \\
\hline Olvido & 2 \\
\hline Presenta reacción adversa & \\
\hline Tapentadol & 3 \\
\hline Dificultad para tomarlo & 1 \\
\hline Incumple voluntariamente & 1 \\
\hline Necesita ayuda & 2 \\
\hline Olvido & 5 \\
\hline Presenta reacción adversa & 1 \\
\hline Se deshacen en el pastillero & \\
\hline
\end{tabular}

p calculada con la t de Student (funcion "PRUEBA.T.N" de Excel).

Tabla 6 Percepción de la eficacia del tratamiento según el propio paciente con dolor no oncológico

\begin{tabular}{|l|c|c|c|c|c|c|c|c|c|}
\hline & \multicolumn{3}{|c|}{ Fentanilo } & \multicolumn{3}{c|}{ Tapentadol } & \multicolumn{3}{c|}{ Total } \\
\cline { 2 - 20 } & $\begin{array}{l}\text { No se les } \\
\text { pregunta }\end{array}$ & No & Si & $\begin{array}{l}\text { No se les } \\
\text { pregunta }\end{array}$ & No & Si & $\begin{array}{c}\text { No se les } \\
\text { pregunta }\end{array}$ & No & Si \\
\hline Controlado el dolor crónico & 23 & 109 & 127 & 44 & 106 & 71 & 67 & 215 & 198 \\
\hline Mejora capacidad funcional & 23 & 81 & 155 & 44 & 77 & 99 & 67 & 158 & 254 \\
\hline Mejora calidad de vida & 23 & 69 & 165 & 44 & 68 & 107 & 67 & 137 & 272 \\
\hline Controlado el dolor crónico (\%) & $9 \%$ & $42 \%$ & $49 \%$ & $20 \%$ & $48 \%$ & $32 \%$ & $14 \%$ & $45 \%$ & $41 \%$ \\
\hline Mejora capacidad funcional (\%) & $9 \%$ & $31 \%$ & $60 \%$ & $20 \%$ & $35 \%$ & $45 \%$ & $14 \%$ & $33 \%$ & $53 \%$ \\
\hline Mejora calidad de vida (\%) & $9 \%$ & $27 \%$ & $64 \%$ & $20 \%$ & $31 \%$ & $49 \%$ & $14 \%$ & $29 \%$ & $57 \%$ \\
\hline
\end{tabular}

No se les pregunta en el caso de los tratamientos de inicio.

Tabla 7 Intervenciones realizadas por los farmacéuticos comunitarios

\begin{tabular}{|l|c|c|c|c|c|c|}
\hline & Fentanilo & Tapentadol & Ambos & Fentanilo & Tapentadol & Ambos \\
\hline Dispensar con IPM & 188 & 131 & 319 & $73 \%$ & $60 \%$ & $66,7 \%$ \\
\hline Dispensar con EPS & 124 & 128 & 252 & $48 \%$ & $58 \%$ & $52,7 \%$ \\
\hline Dispensar y ofrecer SPFA & 45 & 62 & 107 & $17 \%$ & $28 \%$ & $22,4 \%$ \\
\hline Dispensar y derivar & 18 & 14 & 32 & $7 \%$ & $6 \%$ & $6,7 \%$ \\
\hline No dispensar y derivar & 1 & 3 & 4 & $0 \%$ & $1 \%$ & $0,8 \%$ \\
\hline Notificar a SEFV & 0 & 1 & 1 & $0 \%$ & $0 \%$ & $0 \%$ \\
\hline Total pacientes & 258 & 220 & 478 & & & \\
\hline
\end{tabular}

IPM: información personalizada del medicamento; EPS: educación para la salud; SPFA: servicio profesional farmacéutico asistencial; SEFV: Sistema Español de Farmacovigilancia 
Tabla 8 Valoración del interés por un SPFA de abordaje del dolor crónico a los pacientes con dolor no oncológico

\begin{tabular}{|l|c|c|c|c|c|c|}
\hline \multicolumn{1}{|c|}{ Desea SPF } & Fentanilo & Tapentadol & Ambos & Fentanilo & Tapentadol & Ambos \\
\hline Sin datos & 1 & 2 & 3 & - & - & - \\
\hline No & 33 & 14 & 47 & $13 \%$ & $6 \%$ & $9,83 \%$ \\
\hline Ns/Nc & 96 & 74 & 170 & $37 \%$ & $34 \%$ & $35,57 \%$ \\
\hline Si & 128 & 130 & 258 & $50 \%$ & $59 \%$ & $53,98 \%$ \\
\hline Total & 258 & 220 & 478 & & & \\
\hline
\end{tabular}

Los pacientes con tapentadol aprecian más el SPFA $\chi^{2}=7,39 ; p=0,0248$.

A la población del estudio se le preguntó si les gustaría que le ofreciéramos un Servicio Profesional Farmacéutico Asistencial (SPFA) de abordaje del dolor crónico, la respuesta para un 53,9\% fue positiva, y solo el 9,8\% respondió negativamente. Si tenemos en cuenta el tipo de tratamiento, para los pacientes tratados con tapentadol en el $59 \%$ su respuesta fue sí, mientras que para los pacientes en tratamiento con fentanilo fue el $50 \%$ como se refleja en la (tabla 8).

\section{Discusión}

En nuestro estudio realizado sobre el análisis del uso de fentanilo y tapentadol en farmacia comunitaria se puede observar que el $86 \%$ de los pacientes presentaron una indicación no oncológica, de los cuales el 96\% fue por DCNO y solo 4\% para dolor agudo no oncológico.

Nuestros datos reflejan una mayor utilización de opioides para tratar el DCNO frente a la indicación oncológica que supuso un 14\%. Una explicación podemos encontrarla en el análisis que hace el Ministerio de Sanidad (7), donde se expone que el uso de opioides mayores ha experimentado un notable aumento, en gran medida por el incremento de uso para dolor crónico no oncológico.

Observamos también diferencias según sea el sexo del paciente, siendo mayor el número de mujeres que de hombres y en las mujeres es superior el porcentaje de indicación no oncológica respecto de los varones. En mujeres es más frecuente la indicación no oncológica. Nuestros datos coinciden con las cifras de mayor prevalencia del DCNO en las mujeres, como se recoge en la publicación del Ministerio de Sanidad, donde se recopilan todos los estudios de prevalencia del dolor crónico desde el año 2001 (3).

También se observan diferencias en cuanto al medicamento que se emplea según el sexo. Fentanilo se prescribe más a hombres que a mujeres, mientras que a la inversa tapentadol se prescribe más a mujeres que a hombres, aunque las diferencias no llegan a ser significativas $(p=0,0505)$, como se puede ver en la tabla 1 .

En nuestro estudio son los mayores de 70 años los que más prescripciones tienen de estos opioides, con un $55 \%$, seguido de los pacientes de 40 a 69 años con el $42 \%$. Tras analizar el rango de edad con el medicamento que utiliza el paciente se desprende que fentanilo se usa más en mayores de 70 años $(p=0,001)$, como se puede ver en la tabla 1.

La adherencia farmacoterapéutica no resulta fácil de medir y para evaluarla en nuestro estudio se escogió el test de Haynes-Sackett, por ser sencillo y de fácil aplicación. Se trata de un método indirecto de medida que se caracteriza por una elevada especificidad, baja sensibilidad y tiende a infravalorar el incumplimiento. Lo que explica que tan solo el 12,9\% de los pacientes con tratamiento de continuación sí refirieron tener dificultad para tomarse/ponerse su tratamiento (no adherencia).

Sabemos que algunos estudios sitúan la falta de adherencia alrededor del 50\% en el caso de las patologías crónicas. Así, en el estudio Cumplimiento terapéutico: ¿qué conocemos de España?, la mitad de los pacientes con enfermedades crónicas presenta problemas de cumplimiento, aunque éstos descienden hasta el 20\% en las enfermedades reumáticas (artrosis, artritis, etc.) (13). De forma similar en nuestro estudio, la tasa de incumplimiento está por debajo del 50\%, con un valor del $12,9 \%$ de pacientes no adherentes.

Si se tiene en cuenta el tipo de tratamiento que utiliza el paciente, cabe destacar que es mayor la tasa de no adherencia en los pacientes tratados con el principio activo fentanilo, ( $p=0,0039$ ), como se puede ver en la tabla 2.

En nuestro estudio los principales motivos de la no adherencia no fueron los olvidos, ni el incumplimiento voluntario, ni presentar una reacción adversa, sino que fueron ser persona dependiente, tener dificultad en la colocación y tener problemas de adhesión del parche de fentanilo, como lo refleja la tabla 4. Llama la atención que los olvidos, el incumplimiento voluntario y presentar una reacción adversa no suponga mayor tasa de no adherencia, y este hecho puede deberse a que el test de Haynes-Sacket infravalora la falta de adherencia.

Revisando otros estudios que analizan problemas de incumplimiento, en el estudio de Noceda et al (22), el $38,7 \%$ de los pacientes se encuentra sin tratamiento analgésico alguno o lo recibe en dosis inferiores a la dosis diaria definida. Según el análisis de Noceda la situación puede ser debida tanto al mal cumplimiento por parte del paciente como a la baja prescripción por parte de su médico, o a ambos.

Otro de los posibles motivos de falta de adherencia según el análisis del estudio EPIDOR (23), es que la mitad de los pacientes no tiene suficiente confianza en su tratamiento farmacológico para el reuma o no está satisfecho con el tratamiento que recibe para el dolor.

Respecto a los valores EVA globales del estudio, el estudio arroja un promedio de 6,18 puntos de EVA, donde el 44,5\% presenta valores de 
EVA superiores a 6 puntos. Si tenemos en consideración las guías clínicas en DCNO (24) (se considera un tratamiento óptimo aquel que reduzca la EVA en menor o igual al 30\% inicial) podemos constatar que gran parte de los pacientes del estudio no cumplen con los objetivos terapéuticos. Esta afirmación se confirma con otros estudios como la iniciativa Pain Proposal (4), donde el 29\% de los pacientes comenta no tener la sensación de recibir un tratamiento adecuado para su dolor.

En cuanto a los valores obtenidos de EVA en función de la adherencia farmacoterapéutica. Los pacientes no adherentes obtienen un promedio de 6,6 puntos en contraposición a los adherentes con un valor de 6 (tabla 5). Es un valor que indica una tendencia a un menor control del dolor en los pacientes no adherentes. Esto coincide con resultados obtenidos por el Observatorio del Dolor y Timmerman et al (25-26), mostrando la misma tendencia sin que esta tenga relevancia significativa. La no relevancia puede venir dada por aspectos, tales como evaluar la adherencia sin una entrevista estructurada en comparación a estudios que realizan escalas para valorar la intensidad del dolor.

La evaluación media del dolor en los pacientes del estudio fue de 6,1 puntos en la EVA; un 44,5\% de los pacientes presenta valores de EVA superior a 6 puntos.

En cuanto a la percepción de la eficacia del tratamiento para los pacientes con dolor no oncológico (tabla 6), hay que destacar que un 45\% considera no tener el dolor controlado, para un 33\% no ha mejorado su capacidad funcional y para un 29\% no se ha mejorado su calidad de vida. Según el estudio realizado por Pain Alliance Europe, el 50\% de las personas con dolor crónico considera que su calidad de vida se ha reducido a más de la mitad en comparación con el periodo anterior al inicio de su dolor (27).

Además de los problemas de adherencia, las reacciones adversas y los problemas de efectividad en el uso de estos tratamientos suponen un problema en su uso para los pacientes del estudio.

Es recomendable que los FC enseñemos a los pacientes como utilizar los opioides fuertes, siendo la no correcta administración uno de los errores que provoca eventos adversos (7).
Para ello, en nuestro estudio se ofreció a los pacientes información del uso adecuado de estos tratamientos para disminuir las dificultades que tienen en el uso de estos tratamientos y mejorar la adherencia.

La dispensación con información personalizada del medicamento y la educación sanitaria han sido las principales actuaciones del farmacéutico garantizando el conocimiento del adecuado proceso de uso del fentanilo o del tapentadol en la población del estudio, como agente sanitario fundamental en el uso racional de los opioides fuertes, así como contribuyendo en la optimización del tratamiento al resolver los problemas de uso dichos tratamientos.

La alta prevalencia de los pacientes aquejados de DCNO, que acuden a las farmacias comunitarias, justifica el interés profesional por ampliar conocimientos sobre el dolor crónico y los tratamientos efectivos, para poder abordar a estos pacientes adecuadamente. Por ello, el farmacéutico comunitario tiene un papel muy importante en la dispensación de estos medicamentos, garantizando un uso responsable de los mismos y resolviendo problemas de uso que puedan disminuir la adherencia.

Finalmente, en cuanto al interés del paciente por un SPFA, nos llama la atención que un 35\% de pacientes no sabe qué contestar, lo que nos tiene que hacer pensar que no conocen toda la labor asistencial que el farmacéutico comunitario realiza y hay que dar más visibilidad al trabajo que realizamos los farmacéuticos. Como dato, los pacientes con tapentadol aprecian más el SPFA $\chi^{2}=7,39 ; \mathrm{p}=$ 0,0248

Las limitaciones de nuestro trabajo son el tamaño muestral comparado con el elevado número de prescripciones de fentanilo y tapentadol. En los últimos años, ha habido un aumento en la prescripción y dispensación de fármacos opioides fuertes. Fentanilo y tapentadol son un ejemplo de ese aumento (11). Otra limitación de este estudio es emplear un método indirecto para la valoración del cumplimiento cuando no son los más fiables; pero cumple la premisa de ser sencillo y de fácil aplicación, y permite obtener una información que puede ayudar al farmacéutico comunitario a un primer reconocimiento del problema.

\section{Conclusión}

La mayoría de los pacientes del estudio son adherentes al tratamiento. El principal motivo de no adherencia fue el ser persona dependiente, así como los problemas de adhesión y colocación del parche de fentanilo.

Los pacientes del estudio tienen valores EVA promedio de 6 (dolor moderado). Existe una tendencia a tener mayores valores de EVA en pacientes no adherentes.

En cuanto a la percepción de los pacientes, la mayoría de ellos demanda un mayor control del dolor, aunque reconocen tener mejor capacidad funcional y calidad de vida.

Dentro de las incidencias detectadas, un $37,9 \%$ de los pacientes con tratamientos de continuación manifestó tener alguna reacción adversa a fentanilo o tapentadol. El estreñimiento junto con la sedación, en ese orden, fueron las más frecuentes.

Las actuaciones del farmacéutico sirven de ayuda en la optimización del tratamiento, en relación a los problemas de uso de fentanilo y tapentadol, siendo fundamental para mejorar la efectividad y seguridad de ellos.

La mitad de los pacientes estarian interesados en un servicio profesional farmacéutico en dolor crónico. El desarrollo de este servicio permitiría colaborar con el equipo multidisciplinar en la consecución del objetivo que se persiguen con estos tratamientos.

\section{Agradecimientos}

A los 139 farmacéuticos que han colaborado de forma desinteresada en el estudio.

A SEFAC por poner a disposición del grupo de Dolor las plataformas informáticas para llevar a cabo el estudio.

\section{Referencias bibliográficas}

1. López Pérez FJ, Mínguez Martí A, Vicario Sánchez E, Pastor Clérigues A, Sanfeliu García J, Ortega García MP. Experiencia clínica en cambio de opioides en el tratamiento del dolor crónico no oncológico. Farm Hosp (Toledo) 2014; 38(5):411-417. Disponible en: http://scielo.isciii.es/ scielo.php?script=sci_arttextetpi$\mathrm{d}=$ S1130-63432014000500004

2. Doukkali A, Murillo D, Motilva V, Lacalle JR. Intervención farmacéutica y percepción del paciente sobre su dolor. Farmacéuticos Comunitarios 2011 Sep 30; 2(3): 93-99. Disponible en: https:// www.farmaceuticoscomunitarios.org/ 
es/journal-article/intervencion-farmaceutica-percepcion-del-paciente-sobre-su-dolor

3. Andrés J, Acuña JP, Olivares A. Dolor en el paciente de la tercera edad. Rev Med Clin Condes 2014; 25(4): 674-686. doi:10.1016/S0716-8640(14)70089-6

4. Palanca Sánchez I, Puig Riera de Conías MM, Elola Somoza I, Bernal Sobrino JL, Paniagua Caparrós JL. G. Unidad de tratamiento de dolor, estándares y recomendaciones [Monografía en Internet]. Madrid: Ministerio de Sanidad, Politica Social e Igualdad; 2011 [acceso 6 de septiembre de 2018] Disponible en: https://es.calameo. com/read/000072525c316dfec325a

5. Torralba A, Miquel A y Darba J. Situación actual del dolor crónico en España: iniciativa "Pain Proposal”. Rev. Soc. Esp. Dolor 21 (1): 16-22. 2014. doi:10.4321/S113480462014000100003

6. Dueñas M, Salazar A, Ojeda B, Palacín F, Micó JA, Torres LM, Failde I. A Nationwide Study of Chronic Pain Prevalence in the General Spanish Population: Identifying Clinical Subgroups Through Cluster Analysis. Pain Medicine 2015; 16: 811-822. doi:10.1111/ pme. 12640

7. Ministerio de Sanidad, Consumo y Bienestar Social e Instituto Nacional de Estadística (INE). Encuesta Nacional de Salud de España 2017 (ENSE 2017) [Monografía en Internet]. Madrid: Ministerio de Sanidad, Consumo y Bienestar Social; 2017 [acceso 8 de diciembre de 2019] Disponible en: https://www.mscbs.gob.es/estadEstudios/estadisticas/encuestaNacional/ encuestaNac2017/ENSE2017_notatecnica.pdf

8. Prácticas seguras para el uso de opioides en pacientes con dolor crónico [Monografía en Internet]. Madrid: Ministerio de Sanidad, Servicios Sociales e Igualdad; 2015 [acceso 2 de septiembre de 2018] Disponible en: https://www.sedolor.es/download/ practicas-seguras-uso-opioides-pacientes-dolor-cronico/

9. Reuben DB, Alvanzo A, Ashikaga T, Bogat GA, Callahan CM, Ruffing V, et al. National Institutes of Health Pathways to Prevention Workshop: The Role of Opioids in the Treatment of Chronic Pain. Ann Intern Med. 2015; 162(4):295-300. doi:10.7326/M142775

10. Català E, Ferrándiz M, Lorente L, Landaluce Z, Genové M. Uso de opioides en el tratamiento del dolor crónico no oncológico. Recomendaciones basadas en las guías de práctica clínica. Rev Esp Anestesiol Reanim. 2011; 58:283-289. doi:10.1016/S00349356 (11)70063-9

11. Tormo Molina J, Marín Conde L, González Urbano M, Ruiz Pérez de la Blanca M, Robles Martín J, Vivar Simón M. Prescripción de opioides mayores en pacientes con dolor no oncológico: descripción de sus características en una zona de salud de atención primaria. Rev Soc Esp Dolor 2017; 24(1): 19-26. Disponible en: http://scielo.isciii.es/pdf/dolor/v24n1/ original3.pdf

12. Utilización de medicamentos opioides en España durante el periodo 2010-2018 [Monografía en Internet]. Madrid: Agencia Española del Medicamento y Productos Sanitarios; 2019 [acceso 8 de diciembre de 2019] Disponible en: https://www.aemps.gob.es/ medicamentos-de-uso-humano/observatorio-de-uso-de-medicamentos/ utilizacion-de-medicamentos-opioides-en-espana-durante-el-periodo-2010-2018/

13. Rigueira García AI. Cumplimiento terapéutico: ¿qué conocemos de España? Aten Primaria 2001; 27(8): 559-68. doi:10.1016/S0212-6567(01)78860-9

14. Prats Mas $\mathrm{R}$, et al. Dispensación, adherencia y uso adecuado del tratamiento: guía práctica para el farmacéutico comunitario (ADHe+). Barcelona: SEFAC, 2017. Disponible en: https://www.sefac.org/sites/default/ files/2017-11/Adherencia_0.pdf

15. González A, Reyes D, Merencio E, Córcoles M.E. Dispensación de fentanilo y tapentadol en farmacia comunitaria: perfil del paciente e indicación terapéutica en dolor crónico no oncológico. Farmacéuticos Comunitarios. 2018 Sep 28; 10(3):25-31. doi:10.5672/FC. 2173-9218.(2018/Vol10).003.04

16. Sackett DL, Haynes RB, Guyatt GH, Tugwell P. Cumplimiento. Clinical epidemiology: a basic science for clinical medicine. $1^{\text {a }}$ Ed. Boston: Little, 1985.

17. Huskisson EC, Jones J, Scott PJ. Application of visual-analogue scales to the measurement of functional capacity. Rheumatol Rehabil 1976; 15: 185-187. doi:10.1093/rheumatology/ 15.3.185

18. Price DD, McGrath PA, Rafii A, et al. The validation of visual analogue scales as ratio scale measures in chronic and experimental pain. Pain
1983; 17: 45-56 doi:10.1016/03043959(83)90126-4

19. Price DD. Psychological and neural mechanisms of pain. New York: Raven, 1988.

20. Yarnitsky D, Sprecher E, Zaslansky R. Multiple session expenmental pain measurements. Pain 1996; 67: 327-33. doi:10.1016/0304-3959(96) 03110-7

21. Foro de Atención Farmacéutica en Farmacia Comunitaria, panel de expertos. Guía práctica para los servicios de atención farmacéutica en la farmacia comunitaria. Madrid: Consejo General de Colegios Oficiales de Farmacéuticos; 2010. Disponible en: https:// www.portalfarma.com/inicio/atencionfarmaceutica/forofarmaciacomunitaria/Documents/ATFC_Guia\%20 FORO.pdf

22. Noceda Bermejo JJ, Moret Ten C, Lauzirika Alonso I. Tratamiento del dolor osteomuscular crónico. Aten Primaria 2007; 39(1): 29-33. doi:10.1157/13098276

23. Gamero F, Gabriel R, Carbonell J, Tornero J, Sánchez I. El dolor en las consultas de Reumatología españolas: estudio epidemiológico EPIDOR. Rev Clin Esp. 2005; 205:157-63. doi:10.1157/13074161

24. Berrocoso Martínez A, De Arriba Muñoz L, Arcega Baraza A. Abordaje del dolor crónico no oncológico. Rev Clin Med Fam vol.11 no.3 Albacete oct. 2018 Epub 01-Oct-2019. Disponible en: http://scielo.isciii.es/ scielo.php?script=sci_arttextEtpi$d=S 1699-695 X 2018000300154$

25. De Sola H, Salazar A, Dueñas M, Failde I. Opioids in the treatment of pain. Beliefs, knowledge, and attitudes of the general Spanish population. Identification of subgroups through cluster analysis. J Pain Symptom Manage. 2018 Apr; 55(4):1095-1104. doi:10.1016/j. jpainsymman.2017.12.474

26. Timmerman L, Stronks DL, Huygen FJ. The Relation Between Patients, Beliefs About Pain Medication, Medication Adherence, and Treatment Outcome in Chronic Pain Patients: A Prospective Study. Clin J Pain. 2019 Dec;35(12):941-947. doi:10.1097/AJP. 0000000000000760

27. Pain Alliance Europe. Chronic Pain and Stigma. Survey 2019 Chronic Pain and Stigma. Pain Alliance Europe. Results highlights. ; 2019. [acceso 8 de diciembre de 2019] Disponible en: https://pae-eu.eu/ wp-content/uploads/ 2019/09/2019-Survey-PAE-final-short-report.pdf 


\section{ANEXO. PNT estudio 'Análisis del uso de fentanilo y tapentadol en farmacia comunitaria'}

\section{Análisis del uso de FENTANILO Y TAPENTADOL en farmacia comunitaria Procedimiento normalizado de trabajo}

\section{INTRODUCCIÓN}

El dolor constituye una patología de importancia creciente a nivel mundial (1). Su prevalencia aumenta de forma paralela al envejecimiento de la población y cronificación de las enfermedades.

Las cifras de prevalencia de dolor crónico en la población en general se encuentran entre el $15 \%$ y el $25 \%$, cifra que aumenta al $50 \%$ en los mayores de 65 años y en cualquier grupo afecta más a las mujeres que a los hombres. En 2011, el Ministerio de Sanidad y Consumo edita una publicación denominada "Unidad de tratamiento del dolor. Estándares y recomendaciones de calidad y seguridad". En ella, se recopilan todos los estudios de prevalencia del dolor crónico desde el año 2001 (2), reflejando una prevalencia para el dolor crónico del $23,8 \%$, además de, una relación mujer/hombre de 37/20.

Por otro lado, la última encuesta nacional de salud (3) en España (2011-2012) muestra que uno de cada seis adultos encuestados refiere dolor lumbar/cervical crónico o refieren artrosis, dolencias referidas como las principales causas de dolor crónico no oncológico $(4,5)$.

\section{JUSTIFICACIÓN}

Dentro del dolor crónico, el dolor no oncológico supone un reto debido a su complejidad e imprevisibilidad de respuesta al tratamiento. En este tipo de patologías, en la actualidad, es cada vez mayor el uso de opioides. Específicamente, el uso de opioides fuertes o mayores para el tratamiento del dolor no oncológico. Poseen amplia experiencia principalmente en dolores nociceptivos (6), aunque en dolor neuropático, la bibliografía recomienda su uso, la experiencia clínica es menor debido a la variabilidad de tipos de dolor.

La efectividad a largo plazo de los opioides fuertes utilizados en el dolor crónico no oncológico sigue siendo controvertida, ya que la mayoría de los estudios sobre su eficacia no son superiores a 12 semanas y los estudios sobre su seguridad no van más allá de 1 año (7). Por lo que tiene que ser indicado y prescrito con precaución y siempre valorando a cada paciente de forma individual (8).

La efectividad inicial puede desaparecer aun siendo los pacientes adherentes al tratamiento. Al no tener techo de dosis, altas dosificaciones pueden originar efectos adversos propios de su uso y posología. Afectan al SNC y el aparato gastrointestinal (náuseas, vómitos, estreñimiento, mareos, somnolencia y sedación). 
En los últimos años, ha habido un aumento en la prescripción y dispensación de fármacos opioides fuertes. El Fentanilo y el Tapentadol son un ejemplo de ese aumento (9). Tanto es que desde la administración se están realizando programas de inspección para valorar el gran consumo de Fentanilo.

La alta prevalencia de los pacientes aquejados de dolor crónico no oncológico, que acuden a las farmacias comunitarias, justifica el interés profesional por ampliar conocimientos sobre el dolor crónico y los tratamientos efectivos, para poder abordar a estos pacientes adecuadamente. Por ello, el farmacéutico comunitario tiene un papel muy importante en la dispensación de estos medicamentos, garantizando un uso responsable de los mismos.

\section{OBJETIVOS DEL ESTUDIO}

El objetivo principal del estudio consiste en registrar las indicaciones de las prescripciones de los opioides fuertes Tapentadol y Fentanilo.

Como objetivos secundarios se pretende conocer:

- La eficacia de los opioides fuertes Fentanilo y Tapentadol en dolor crónico no oncológico.

- Conocer el porcentaje de Fentanilo y Tapentadol prescrito para dolor crónico no oncológico y para dolor oncológico.

- Hacer un seguimiento de la seguridad de los opioides fuertes Tapentadol y Fentanilo.

- Registrar la intervención del farmacéutico en la dispensación de los opioides fuertes Tapentadol y Fentanilo.

\section{OBJETIVOS DEL PROCEDIMIENTO NORMALIZADO DE TRABAJO (PNT)}

Este PNT tiene como objetivo principal servir de guía a todos los farmacéuticos participantes. Facilitando su desarrollo, así como una normalización entre todos los investigadores a la hora de la recogida de datos mediante un cuestionario específicamente elaborado para el estudio.

También se pretende recordar algunas cuestiones intrínsecas a la dispensación de los fármacos que contengan los principios activos Fentanilo o Tapentadol en diferentes materias. Ya sean recomendaciones con respecto al uso adecuado de los medicamentos, normas de educación sanitaria en pacientes no oncológicos, y, por último, comunicación a otros profesionales o instituciones por resultados negativos asociados a la medicación (RNM), etc. 


\section{PROCEDIMIENTO}

\section{Consideraciones previas a la aplicación del cuestionario}

Deberá hacerse en el momento de la dispensación de uno o varios fármacos que contengan Fentanilo o Tapentadol como principios activos, ya sea para la persona que se lo lleva como al cuidador de ésta, encargado de recoger su medicación.

Este estudio queda reservado a los mayores de 18 años que voluntariamente quieran participar. Incidir en que la participación es totalmente anónima y no debería retrasar el proceso de dispensación habitual.

Es recomendable que los farmacéuticos investigadores dispongan del cuestionario en formato electrónico. Este formato facilitará su almacenamiento, agilizará la ejecución del cuestionario con el paciente y además, es una forma de prevención ante posibles extravíos tradicionalmente asociados al uso del formato en papel.

\section{Realización del cuestionario}

En este apartado se expondrán las diferentes cuestiones de las que se compone el cuestionario y qué aspectos debemos tener en cuenta en cada punto o pregunta. Posibilitará una homogenización durante el desarrollo de la entrevista al paciente entre los distintos investigadores farmacéuticos.

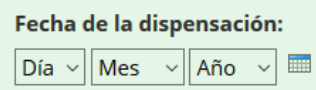

Paciente:

Hombre $\bigcirc$ Mujer
Edad

(18 a 39 años)

(40 a 69 años)

(>70 años)
Responde cuestionario:

O Paciente

O Familiar/Cuidador/a

En primer lugar, anotaremos la fecha de dispensación. A continuación, preguntaremos a nuestro paciente si la medicación a dispensar es para ella o para algún familiar o persona a su cargo. De esta manera evitamos un error durante la anotación del sexo del paciente y podremos, además, anotar el campo referido a quién "responde cuestionario". Por último, preguntaremos al paciente o familiar/ cuidador sobre su edad. 


\section{Medicamento a dispensar:}

G FENTANILO OTAPENTADOL

FENTANILO

$\square$ Transdérmica

$\square$ Transmucosa

$\square$ Bucal

$\square$ Intranasal
OFENTANILO OTAPENTADOL

Dosis (mg)

Dosis (mg)

(20)

Durante el momento de la cuestión "medicamento a dispensar", el farmacéutico deberá cerciorarse de que alguno de los medicamentos a dispensar contiene alguno de los principios activos a estudiar: Fentanilo, Tapentadol o los dos, en caso de dispensar dos fármacos o más. Se comprobará si el fármaco es el correcto a través de la receta que adjunte el paciente ya sea en formato electrónico o en papel.

También debe anotarse la dosificación del fármaco y su forma de liberación. En caso de contener Fentanilo se anotará la dosis expresada en microgramos y su forma de liberación entre las distintas opciones en las que puede presentarse el fármaco. En el caso de los fármacos de contengan Tapentadol, expresaremos la dosis en miligramos y no tendremos que expresar su presentación debido a que el Tapentadol sólo se presenta a través de comprimidos de liberación gradual. 
Indicación terapéutica:

O. ONCOLÓGICA

Has finalizado el cuestionario.

Pulsa sobre el botón "Enviar"

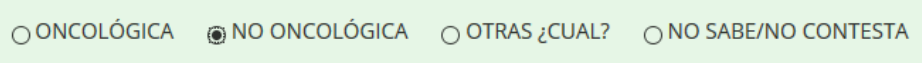

NO ONCOLÓGICA

$\square$ Dolor crónico

$\square$ Lumbalgia

$\square$ Artrosis

$\square$ Otras ¿cuál?

Durante el desarrollo de este apartado, preguntaremos al paciente por la patología que manifiesta. Tendremos dos opciones: "oncológica" (puede que el paciente no sepa su significado, en ese caso hablar de cáncer) o "no oncológica" en caso de que la primera opción sea negativa. En caso de estar en presencia de un paciente oncológico, marcaremos su respuesta y habremos finalizado el cuestionario.

En caso de que el paciente sea no oncológico. Deberemos profundizar en qué tipo de patología tiene. Podemos diferenciar entre "Lumbalgia, Artrosis, Dolor crónico" o en caso de no reunir los criterios para esas patologías, tenemos la opción "Otras", también, en su defecto, si el paciente la desconoce o no quiere comentarla, tenemos la opción No sabe/no contesta.

Tenemos que tener en cuenta que a veces nuestros pacientes suelen confundir su diagnóstico. Normalmente, el error más probable es la diferencia entre artrosis y artritis. Para asegurarnos de que estamos marcando la opción correcta, tengamos en cuenta que la artrosis y la artritis pueden parecer patologías con sintomatología similar, pero en el caso de la artrosis, esta suele empeorar con el esfuerzo y movimiento, y mejorar con un reposo breve, cosa que en el caso de la artritis al tener una etiología inflamatoria no mejora con el reposo.

Por último, tener en cuenta que sólo la variable "Dolor oncológico" hará que finalicemos la entrevista con el paciente.

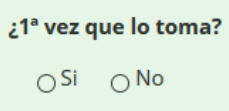

$$
\mathrm{OSi} O \mathrm{No}
$$

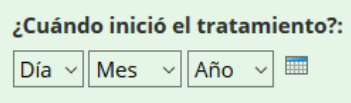

¿Ha respondido alguna vez este

cuestionario?

OSi ONo ONs/Nc

Con estas cuestiones evaluaremos varios parámetros, si el paciente se lo toma por primera vez, en caso contrario, anotaremos el inicio del tratamiento, siempre utilizando como medida semanas, meses o años.

Está permitido que el paciente pueda responder más de una vez al cuestionario. Medir esta variable nos permitirá describir la evolución de los pacientes durante el tiempo. Si el paciente no recuerda si lo ha contestado alguna vez, tenemos habilitada la opción Ns/Nc. 


Sufre Reacción adversa
ONo $\quad \mathrm{Si}$
¿Qué reacción adversa sufre?
$\square$ Estreñimiento
$\square$ Sedación
$\square$ Nauseas y/o vómitos
$\square$ Prurito
$\square$ Otras (especificar):

Estos parámetros del cuestionario sólo serán rellenados por el investigador en caso de que no sea la primera vez que el paciente utiliza el fármaco.

Mediremos las posibles reacciones adversas que pueda haber experimentado el paciente con el uso del medicamento. Es importante que a la hora de desarrollar este ítem no nombremos ninguna de las reacciones adversas para no condicionar la respuesta del paciente. Podemos utilizar frases como: ¿qué tal le sienta el medicamento?, ¿ha experimentado algún efecto extraño? o ¿asocia el efecto con la toma del fármaco?

En caso afirmativo de reacción adversa y no sea de las clásicas reacciones de los opioides habilitadas, marcar la opción "Otras" y escribir lo más preciso posible su efecto.

\section{ADHERENCIA}

La mayoría de la gente tiene dificultad en tomarse/ponerse los medicamentos, ¿tiene dificultad en tomarse/ponerse los suyos? No $\mathrm{OSi}$

¿Qué dificultad tiene para tomarse o ponerse los medicamentos?

¿Cómo se los ha tomado/puesto?

OBien $\mathrm{Mal}$

Aquí trataremos la adherencia del paciente a su tratamiento y uso de él, el paciente contestará a estas preguntas siempre que no sea la primera vez que usa el fármaco.

Intentar incluir estas cuestiones en un diálogo fluido, nos permitirá una complicidad con la persona a estudio y disminuir la sensación del individuo de ser juzgado.

Intentar ser lo más específico posible a la hora de describir la dificultad a la hora del uso del medicamento (2a pregunta). 


\section{Posología ¿Cómo toma/usa su medicación? ¿Cada cuanto?}

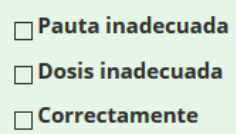

En el desarrollo de esta parte del cuestionario, debemos tener en cuenta que no contestarán estas preguntas aquellos pacientes que usen por primera vez su medicación.

Se evalúa si el paciente está tomando bien su medicación o no. En caso contrario, se especificará los motivos por los que el paciente no cumple, ya sea por no cumplir con la pauta indicada o por no tomar la dosis correcta. Comprobar los datos de posología mediante la receta en formato electrónico o papel con lo que el paciente nos cuenta.

Es importante, como en el apartado anterior comentábamos, incorporar estas preguntas en un diálogo fluido para evitar que el paciente tenga la sensación de ser juzgado.

Al finalizar este diálogo debemos ser capaces de contestar si el paciente presenta algún PRM (Problema Relacionado con el Medicamento) y algún RNM (Resultado Negativo a la Medicación). 
¿Ha detectado algún PRM? (Problema Relacionado con el Medicamento) *

NO • sí

En caso de ser Sí se nos debería abrir esta lista y poder marcar una o más opciones:

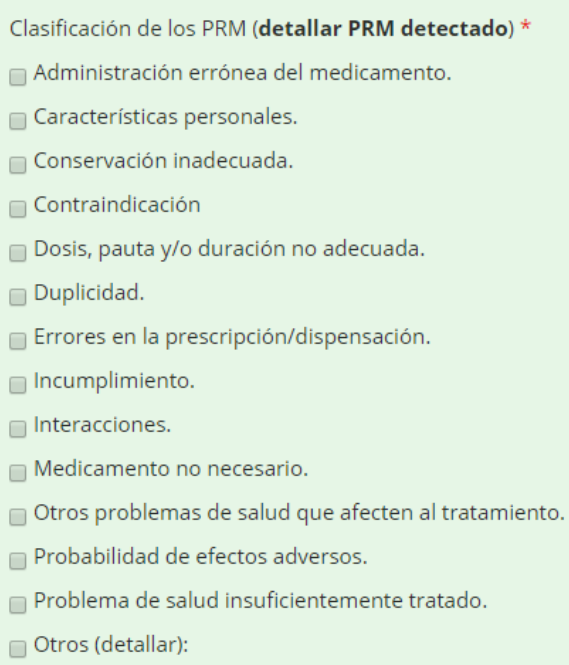


¿Ha detectado algún RNM? (Resultado Negativo a la medicación) *

○

En caso de ser Sí se nos debería abrir esta lista y poder marcar una o más opciones:

Clasificación de los RNM (detallar RNM detectado) *

$\square$ Necesidad de medicamento (problema de salud no tratado). El paciente sufre un problema de salud asociado a no recibir un medicamento que necesita.

No necesidad de medicamento (efecto de medicamento innecesario). El paciente sufre un problema de salud asociado a recibir un medicamento que no necesita.

$\square$ Inefectividad no cuantitativa. El paciente sufre un problema de salud asociado a una inefectividad no cuantitativa de la medicación.

$\square$ Inefectividad cuantitativa. El paciente sufre un problema de salud asociado a una inefectividad cuantitativa de la medicación.

Inseguridad no cuantitativa. El paciente sufre un problema de salud asociado a una inseguridad no cuantitativa de un medicamento.

$\square$ Inseguridad cuantitativa. El paciente sufre un problema de salud asociado a una inseguridad cuantitativa de un medicamento. 


\section{Características del dolor}

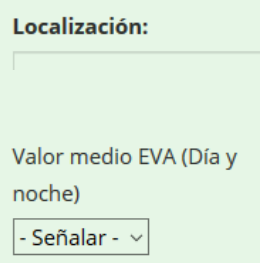

Causa:

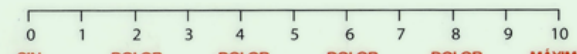

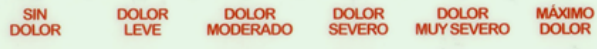

- Señalar - $\checkmark$

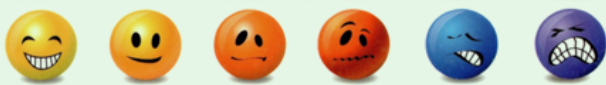

Las características del dolor también son objetivo del cuestionario. Deberemos rellenar varios campos de estudio:

Localización: especificar la región anatómica donde se sitúa el dolor.

Causa: especificaremos la razón del dolor. Ejemplos: contractura muscular, inflamación, etc.

Valor medio escala EVA: escala clásica de evaluación del dolor, se define como una línea recta en la que un extremo significa ausencia de dolor y el otro extremo significa el peor dolor que se pueda imaginar. El paciente marca un punto en la línea que coincide con la cantidad de dolor que siente. Para evaluar, haremos la siguiente pregunta al paciente: En una escala de 0 a 10, siendo 0 ausencia de dolor y 10 el peor dolor que pueda imaginarse, ¿cuánto le duele de media a lo largo del día? A continuación, apuntaremos el valor que nos indique el paciente.

Es importante que en la valoración del dolor, cuando hablemos al paciente sobre 10 en la escala sea del peor dolor que pueda imaginarse, no del que recuerda, ya que estaríamos cometiendo un sesgo. 


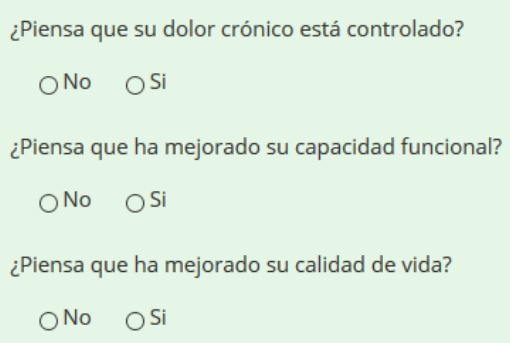

Estos dos apartados del cuestionario son posibles de realizar seguidamente ya que nos hablan de la opinión personal del paciente con respecto a la eficacia y función de su tratamiento. Siempre y cuando no sea la primera vez que toma la medicación, en este caso sólo el segundo apartado.

Como consideraciones especiales, puede que el paciente en la 2a pregunta no entienda, en su totalidad, el significado de capacidad funcional, para ello, podemos adaptar la pregunta a ¿piensa que ha mejorado su capacidad para realizar tareas diarias? Por otro lado, tener en cuenta que las 3 primeras preguntas se contestan con un No o Sí, no como la pregunta 4 en la que el paciente puede elegir varias de las opciones disponibles. 


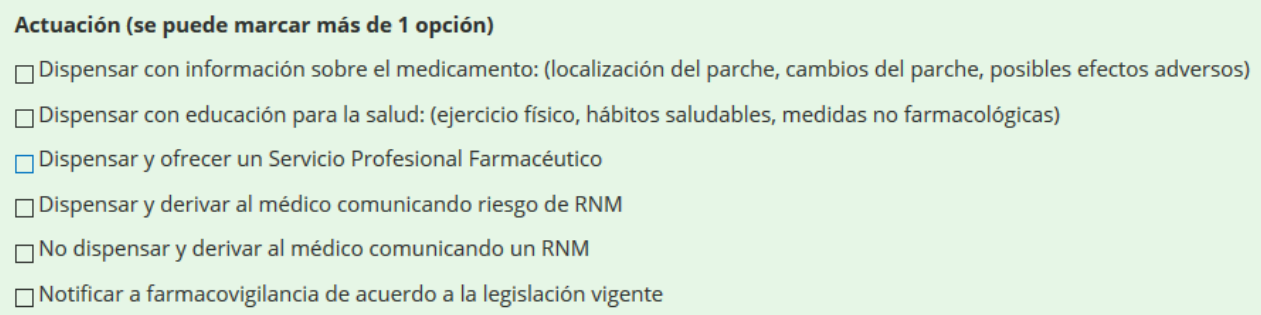

El desarrollo de este apartado consistirá en dar al paciente información adicional al paciente sobre su tratamiento, recomendaciones sobre su patología crónica o la comunicación de diversos aspectos de alarma en relación a su tratamiento a otros profesionales sanitarios.

Es posible por parte del investigador realizar más de una de las opciones disponibles. Comentaremos cada una de ellas:

Dispensar con información sobre el medicamento

A través del Anexo 1 se pondrán a disposición del farmacéutico información acerca del uso y control de los principios activos a estudio, así como sus distintas formas de liberación. Puede servirnos para este apartado, así como otros anteriores del cuestionario.

Dispensar con educación para la salud

A través del Anexo 2 se pondrán a disposición del farmacéutico distintos hábitos de vida saludables para el paciente con dolor no oncológico.

Dispensar y ofrecer un Servicio Profesional Farmacéutico

Opción disponible en caso de que el farmacéutico detecte algún problema en el paciente que pueda ser abordable a través de un Servicio Profesional disponible en la farmacia comunitaria.

Dispensar y derivar al médico comunicando riesgo de RNM; No dispensar y Derivar al médico comunicando un RNM

Para ello, se realizará mediante la metodología a elección por el farmacéutico. A modo de ayuda, se recomienda la "Guía Práctica para los Servicios de Atención Farmacéutica en la Farmacia Comunitaria" publicada por el Foro de Atención Farmacéutica para la Farmacia Comunitaria. Puede consultarse desde el siguiente enlace:

http://www.pharmaceutical-care.org/archivos/812/cuaderno_foro-v17_56_pag_alta.pdf

Notificar a farmacovigilancia de acuerdo a la legislación vigente

En caso de que el investigador considere necesario notificar alguna reacción adversa a través del sistema español de farmacovigilancia. Desde este enlace de la AEMPS se describe todo el proceso en caso de duda:

https://www.aemps.gob.es/vigilancia/medicamentosUsoHumano/SEFV-H/NRA-SEFV-H/notifica SospechasRAM-profSanitarios.htm 
¿Le gustaría que le ofreciéramos un Servicio Profesional farmacéutico de abordaje del dolor crónico?

$$
\text { ONo OSi ONo sabe/No Contesta }
$$

Por último, esta pregunta queda reservada ante la posibilidad de creación de un Servicio Profesional en dolor crónico. Con ella, se obtendrá la opinión del paciente diana. 


\section{BIBLIOGRAFÍA}

1. López-Pérez F.J, Mínguez-Martí A, Vicario-Sánchez E, Pastor-Clérigues A, Sanfeliu García J, Ortega-García M.P. Experiencia clínica en cambio de opioides en el tratamiento del dolor crónico no oncológico.Farm Hosp. 2014; 38(5):411-417. DOI: 10.7399/FH.2014.38.5.7549

2. Palanca Sanchez I, Puig riera de Conías MM, Elola Somoza I, Bernal Sobrino JL,Paniagua Caparrós JL,Grupo de expertos. Unidad de tratamiento de dolor, estándares y recomendaciones. Madrid: Ministerio de Sanidad, Politica Social e Igualdad;2011.

3. Encuesta Nacional de Salud 2011-2012. Ministerio de Sanidad. Disponible en: https://www.mscbs.gob.es/ estadEstudios/estadisticas/encuestaNacional/encuesta2011.htm

4. Dobscha SK, Morasco BJ, Duckart JP, Macey T, Deyo RA. Correlates of prescription opioid initiation and long-term opioid use in veterans with persistent pain. Clin J Pain 2013; 29(2):102-8. DOI: 10.1097/AJP.0b013e3182490bdb

5. Reid MC, Henderson CR Jr, Papaleontiou M, Amanfo L, Olkhovskaya Y, Moore AA, et al. Characteristics of older adults receiving opioids in primary care: Treatment duration and outcomes. Pain Med 2010; 11(7):1063-71. DOI: 10.1111/j.1526-4637.2010.00883.x

6. Sociedad Española del Dolor. Grupo de Opioides. Guía clínica de la utilización de opioides en dolor crónico no oncológico.

7. David B. Reuben, Anika A.H. Alvanzo, Takamaru Ashikaga, G. Anne Bogat, Christopher M. Callahan, Victoria Ruffing, et al. National Institutes of Health Pathways to Prevention Workshop: The Role of Opioids in the Treatment of Chronic Pain. Ann Intern Med. 2015;162:295-300. DOI: 10.7326/M14-2775

8. Català E, Ferrándiz M, Lorente L, Landaluce Z, Genové M. Uso de opioides en el tratamiento del dolor crónico no oncológico. Recomendaciones basadas en las guías de práctica clínica. Rev Esp Anestesiol Reanim. 2011; 58:283-289

9. Tormo Molina J, Marín Conde L, González Urbano M, Ruiz Pérez de la Blanca M, Robles Martín J y Vivar Simón M. Prescripción de opioides mayores en pacientes con dolor no oncológico: descripción de sus características en una zona de salud de atención primaria. Rev Soc Esp Dolor 2017; 24(1): 19-26. DOI: 10.20986/resed.2016.3471/2016 


\section{ANEXO 1 \\ Principios activos y formas de liberación CONSIDERACIONES ESPECIALES}

\section{Fentanilo transdérmico}

Este tipo de liberación la permite su liposolubilidad, su efecto no irritante y su bajo peso molecular. El intervalo de dosis es generalmente de 72 horas (retirada del parche), aunque algunos pacientes requieren dosificación cada 48 horas.

- Aplicar en piel no pilosa ni con escoriaciones (no depilar la zona para aplicación).

- No cortar el parche.

- No exponerlo al sol.

- Rotar las zonas cutáneas de aplicación. A las 48 o 72 horas (habitualmente 72 h) sustituir el parche.

- En caso de prurito intenso o eritema, valorar la posibilidad de otra forma de liberación.

- Tras suspenderlo, su efecto puede durar todavía 12-24 horas.

\section{Fentanilo bucal}

Debe utilizarse por vía sublingual. Esto significa que deberá colocarse el comprimido debajo de la lengua, donde se disolverá rápidamente para permitir que se absorba a través del revestimiento de la boca.

Colocar el comprimido bajo la lengua lo más atrás que pueda el paciente, y dejarlo disolver por completo. Es importante no chupar, masticar ni tragar el comprimido. No beber ni comer nada hasta que el comprimido se haya disuelto por completo bajo la lengua.

\section{Fentanilo transmucosa}

Se trata de citrato de fentanilo, que produce un rápido comienzo de acción ( 5 minutos) de corta duración (2,5-5 horas) y de fácil utilización. El aplicador ("chupa-chups") debe ser consumido en 15 minutos. No debe masticarse ni tragarse.

Precaución en pacientes diabéticos por su contenido en glucosa.

\section{Fentanilo intranasal}

Antes de utilizar fentanilo nasal por primera vez, se deberá preparar el pulverizador hasta que aparezca una vaporización fina (normalmente se necesitan de 3 a 4 pulsaciones del pulverizador nasal). Si el producto no se ha utilizado durante un periodo de más de 7 días, se debe pulsar el pulverizador nasal una vez para eliminar el producto sobrante antes de administrar la siguiente dosis.

Se recomienda que el paciente esté sentado durante la administración, principalmente para evitar mareos. 
Antes de su utilización, se debe extraer el frasco del recipiente con cierre de seguridad y quitar el tapón protector. Cebar el frasco antes del primer uso: sostener el frasco en posición vertical y oprimir y soltar los agarres digitales en ambos lados de la boquilla, hasta que aparezca una barra verde en la ventana de recuento (debe aparecer después de cuatro pulverizaciones). Para confirmar que se ha administrado una pulverización, se escuchará un clic o bien el número avanza en el contador.

Se debe aconsejar a los pacientes que no se suenen la nariz inmediatamente después de la administración.

Después de cada utilización es necesario limpiar el extremo del pulverizador nasal.

Habrá que esperar al menos $4 \mathrm{~h}$ antes de tratar con fentanilo nasal otro episodio de dolor irruptivo.

Si pasan cinco días sin que se use el producto, se debe volver a cebar pulverizando una vez. Desechar el frasco 60 días después de la primera apertura.

Presencia de afecciones nasales: si el paciente experimenta episodios repetidos de epistaxis, rinorrea o molestias nasales durante el tratamiento, se deberá considerar una vía de administración alternativa para tratar el dolor irruptivo.

\section{Tapentadol}

Se presenta en comprimidos de liberación gradual. Tragar con abundante agua. Puede tomarse con o sin alimentos.

No produce interacciones a través del citocromo P-450. 
ANEXO 2

\section{Hábitos de vida saludables para el paciente con dolor crónico no oncológico}

\section{Ejercicio físico}

La adecuada actividad física regular de intensidad moderada, acumulando por lo menos 30 minutos casi todos los días de la semana, y el ejercicio como caminar, correr, ejercicios aeróbicos, ejercicio en bicicleta y piscina, pueden mejorar la fuerza muscular y la resistencia, la amplitud de movimiento, el equilibrio, la capacidad aeróbica y la calidad de vida. Pueden reducir el dolor, la fatiga, la depresión y el peso corporal en personas con osteoartrosis, así como en las personas con dolor crónico generalizado y dolor lumbar crónico.

\section{Higiene del sueño}

- Un descanso adecuado mejora la percepción del paciente disminuyendo su ansiedad y por lo tanto la intensidad de su dolor. Normas para tener una higiene del sueño óptima:

- Irse a la cama sólo cuando se tenga sueño y realizar rutinas para inducir el sueño (leer, relajarse...).

- Levantarse y acostarse. Evitar quedarse en cama despierto más tiempo del necesario todos los días a la misma hora, incluido los fines de semana y vacaciones, esquema de tiempo regular para las comidas.

- Evitar quedarse en cama despierto más tiempo del necesario, utilizar el dormitorio solo para dormir.

- Evitar las siestas (no a partir de las $15 \mathrm{~h}$, y breves 15 minutos).

- Reducir o evitar el consumo de alcohol, cafeína y tabaco al menos 6 horas antes de la hora de acostarse.

- Evitar las comidas copiosas antes de acostarse.

- Evite los hipnóticos y sedantes o utilizarlos cuidadosamente, no más de 4 semanas y a la dosis más baja posible.

- Mantener condiciones ambientales adecuadas para dormir (temperatura fresca, ventilación, sin ruidos, oscuridad).

- Evitar actividades estresantes en las horas previas de acostarse.

- Debe realizar diariamente ejercicio físico moderado, preferible por la mañana, debe evitar realizar ejercicio intenso seis horas antes de la hora de acostarse.

- Practicar ejercicios de relajación antes de acostarse.

- Tomar baños de agua a temperatura corporal por su efecto relajante.

\section{Disminuir el estrés}

El estilo de vida occidental y, por consiguiente, el alto nivel de estrés al que actualmente estamos sometidos hacen que la espalda pueda generar cambios degenerativos a nivel osteomuscular. Por ello, se recomiendan las técnicas de relajación y evitar situaciones de alto grado de ansiedad. 


\section{Hábitos dietéticos}

Fomentar el consumo de alimentos ricos en sustancias básicas para el mantenimiento del sistema neuromuscular. Los frutos secos son fuente de omega-3 y calcio, los lácteos tienen gran aporte de calcio y los pescados azules se componen de los omega-3 EPA y DHA, siendo sustancias que forman las membranas celulares y, además, juegan un papel proinflamatorio. Por último, las legumbres, los cereales y los productos de origen animal suministran vitaminas del grupo B.

\section{Carga de peso}

Evitar el exceso de peso lo máximo posible. En caso de hacerlo, mantener una postura óptima.

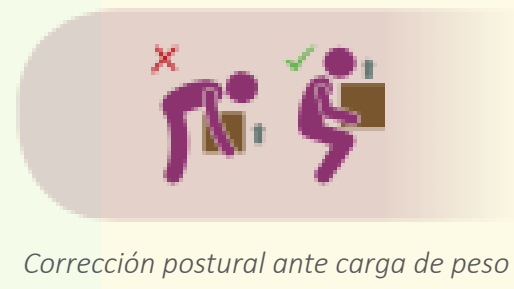

\section{Control del peso}

El exceso de peso puede llevar a un sobresfuerzo de los componentes que componen la espalda, causando sintomatología degenerativa, agravando el dolor o favoreciendo su aparición.

\section{Trabajadores o personas que permanecen mucho tiempo sentadas}

Recomendar el uso de una silla confortable, una mesa de altura adecuada e higiene postural, evitando trabajar con la espalda encorvada.

\section{Alcohol y tabaco}

Se debe evitar las grandes cantidades de alcohol, así como su consumo constante pudiendo producir deficiencias vitamínicas como la B12 que promueven la neuropatía alcohólica. Además, el consumo de tabaco no es recomendable ya que puede agravar patologías que cursan con dolor como las reumatológicas. 